\title{
RESEARCH
}

Open Access

\section{Mat Pilates is as effective as aquatic aerobic exercise in treating women with fibromyalgia: a clinical, randomized and blind trial}

Suzy Araújo de Medeiros ${ }^{1}$, Hugo Jário de Almeida Silva², Rayssa Maria do Nascimento ${ }^{1}$, Jaely Beatriz da Silva Maia ${ }^{1}$, Caio Alano de Almeida Lins ${ }^{2}$ and Marcelo Cardoso de Souza ${ }^{2 *}$ (D)

\begin{abstract}
Background: The mat Pilates method is the therapeutic modalities which can be used in fibromyalgia treatment. Although there are no well-designed studies that prove the effectiveness of the mat Pilates method in this population. The objective was to evaluate the effectiveness of the mat Pilates method for improving symptoms in women with fibromyalgia.
\end{abstract}

Methods: A single blind randomized controlled trial in which 42 women with fibromyalgia were randomized into two groups: mat Pilates and aquatic aerobic exercise. The exercises were performed twice a week for 12 weeks. Two evaluations were performed: one at baseline (TO), and another at 12 weeks after randomization (T12). The primary outcome was pain measured by the Visual Analogue Scale (VAS). Secondary outcomes were function (Fibromyalgia Impact Questionnaire), sleep (Pittsburgh Sleep Quality Index [PSQI]), quality of life (Short Form 36 [SF-36]), fear avoidance (Fear Avoidance Beliefs Questionnaire [FABQ-BR]) and pain catastrophizing (Pain-Related Catastrophizing Thoughts Scale [PRCTS]).

Results: There was improvement in both groups in relation to pain and function $(p<0.05)$. The aspects related to quality of life and the FABQ questionnaire only showed improvement in the mat Pilates group $(p<0.05)$. There was improvement in the PSQI and PRCTS variables only in the aquatic aerobic exercise group $(p<0.05)$, but no differences were observed between the groups for any of the evaluated variables.

Conclusion: Significant improvements were observed in the two groups in relation to the disease symptoms, and no differences were observed between mat Pilates and aquatic aerobic exercise in any of the measured variables.

Trial registration: ClinicalTrials.gov Identifier (NCT03149198), May 11, 2017. Approved by the Ethics Committee of FACISA/UFRN (Number: 2.116.314).

Keywords: Physiotherapy, Aerobic exercise, Rheumatology, Chronic pain

\footnotetext{
* Correspondence: marcelocardoso@facisa.ufrn.br

${ }^{2}$ Federal University of Rio Grande do Norte, Faculty of Health Sciences of

Trairi - (UFRN/FACISA), Postgraduate Program in Rehabilitation Sciences, Vila

Trairi st, S/N, Centro, Santa Cruz, RN 59200-000, Brazil

Full list of author information is available at the end of the article
}

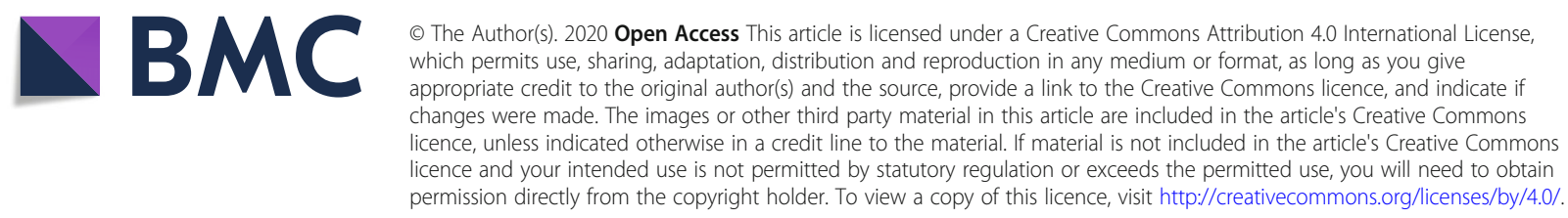




\section{Bullet points}

Mat Pilates improves pain and function in women with fibromyalgia as well as aquatic aerobic exercise.

Mat Pilates can be considered as a treatment option for the symptoms of women with fibromyalgia.

Patient preferences should be taken into account when prescribing exercise.

\section{Background}

Fibromyalgia (FM) is a chronic disease characterized by generalized pain ${ }^{1}$. It is triggered due to peripheral and central hyperexcitability mechanisms which are able to generate alterations in the perception of pain such as hyperalgesia and allodyne, muscular rigidity, reduced functional capacity and sleep alterations [1-6]. FM has a general prevalence ranging from 0.66 to $4.4 \%$, and a prevalence ratio of 8 women for each man, with the most frequent diagnosis occurring in middle age [7].

FM has controversial etiopathogenesis, and its cause is associated with genetic, environmental and neuromodulatory factors [8]. Many patients with fibromyalgia have high levels of stress and feelings of depression, anxiety and frustration [9].

Thus, in addition to pharmacological treatment and educational programs, evidence has been observed over the years regarding treatment with physical exercise [10], in which an effective treatment program consists of stretching, strength maintenance and aerobic conditioning [2], with the main objective to repair the effects of a lack of physical conditioning and to improve the symptoms, especially those of pain and fatigue. Studies reported that physical exercises are beneficial, low cost and promote improvement in pain and other symptoms of fibromyalgia [11-13].

Although studies show benefits for almost all exercise modalities, further evidence supports the practice of aerobic exercise $[14,15]$. Within this context, aquatic aerobic exercise is considered a treatment form with good results for patients with FM. In addition to the specific benefits of physical exercise, the properties of heated water make the aquatic environment a suitable place for performing exercises with the least possible harm to the musculoskeletal system. It provides reduced joint impact, improved microcirculation, facilitates relaxation, decreases the number of contractions and improves muscle strength due to its natural resistance [3, 16, 17]. A Cochrane review showed that aerobic water exercise for FM is effective in improving overall well-being, functional capacity, pain, improved muscle strength by $37 \%$ and improved cardiovascular capacity [18].

The Pilates method has gained popularity for some time. It is an exercise approach founded on the teachings of Joseph Pilates (1880-1967), which aims at improving general body flexibility, core strength, posture and coordination of breathing with movement [19]. The main feature is its six basic principles: centralization, concentration, control, precision, respiration and flow [20]. This modality of therapeutic exercise is well recommended for rehabilitating the musculoskeletal system [21-23], but there are no welldesigned studies demonstrating its benefit in treating fibromyalgia [2].

Even taking into consideration that the Pilates method is widely recommended and prescribed by health professionals, few studies have evaluated the effects of the method to treat patients with FM. These studies present several methodological flaws which compromise their results and suggest that studies with better methodological design be carried out with the objective of observing the effectiveness of the Pilates method in treating FM symptoms $[2,24]$. A study recently compared tai chi with aerobic exercise and showed that tai chi was more effective than aerobic exercise. This study suggested that mindbody exercises may be the best option for patients with fibromyalgia [25]. Within this context, mat Pilates may also be considered an exercise modality that has similar characteristics, which would benefit the studied population.

Therefore, the main objective of this study is to evaluate the effectiveness of the mat Pilates method on improving symptoms in women with fibromyalgia. Our hypothesis is that the mat Pilates method can bring more benefits to improve the disease-related symptoms than that of aquatic aerobic exercise. We chose aquatic aerobic exercise for the purpose of comparison in knowing that aquatic aerobic exercise is a strongly recommended modality as a non-pharmacological treatment for people with fibromyalgia [26]; moreover, we believe that this type of exercise can be considered more fun, providing greater adherence to treatment and few studies have used aquatic aerobic exercises to compare with other exercise modalities in treating fibromyalgia.

\section{Methods}

A single-blind randomized controlled trial was conducted according to the CONSORT (Consolidated Standards of Reporting Trials) [27]. This study was registered (ClinicalTrials.gov Identifier: -..--..--.--) and follows the TIDieR (Template for Intervention Description and Replication) checklist [28]. The study protocol and details of the exercise programs of the two groups were previously published [29].

\section{Setting and participants}

Forty-two (42) women with FM diagnosis were selected according to the 2010 American College of Rheumatology classification criteria [30], between 18 and 60 years of age and with pain between 3 and 8 on the Visual Analogue Pain Scale (VAS) from the waiting list of patients of the 
Clinic Physiotherapy School and Basic Health Units of the city. All women had medical referrals from local rheumatologists who confirmed the disease.

Women with uncontrolled hypertension, decompensated cardiorespiratory disease, history of exerciseinduced syncope or arrhythmias, decompensated diabetes, severe psychiatric illness, history of regular exercise (at least twice a week) in the last 6 months or any another condition that made the patient unable to perform physical exercises were excluded.

\section{Sample size calculation}

The sample size was calculated based on the pain variable to find a difference of \pm 2.0 points between the intervention groups on the VAS [31], with a standard deviation of 2.5 points [32]. A sample of 42 participants was necessary to reach a statistical power of $80 \%$ with a $5 \%$ alpha, allocating 21 into each group. The sample calculation was performed using Gpower3.1 software.

\section{Randomization, concealed allocation and blinding}

The randomization was performed through the website www.randomization.com. An independent researcher who did not participate in any other study procedures performed the randomization process.

After the initial evaluation, the participants were referred to the physiotherapist in charge of the intervention and were allocated to one of two groups by means of simple randomization using opaque and sequentially numbered sealed envelopes in order to maintain allocation secrecy.

The intervention groups were: Mat Pilates group (MPG; $n=21$ ) and Aquatic Aerobic Exercise Group (AAEG; $n=21$ ). The two groups were submitted to treatments twice a week for 12 weeks. The flow diagram of the study is summarized in Fig. 1.

The researchers responsible for the interventions were blinded to the participants' initial assessments, and the researcher responsible for evaluations was blinded to the group allocation.

In addition, data collected during participant evaluations were not disclosed to investigators responsible for interventions, and participants were instructed to not disclose their experience or information related to the intervention.

Finally, the researcher responsible for statistical analysis performed the analysis blindly. Once the intervention was completed, they received an Excel data table with all the necessary data without identification of the subjects or the groups.

\section{Interventions}

\section{Mat Pilates group (MPG)}

The MPG patients performed exercises based on the Mat Pilates method in a group of up to 4 women in a large and comfortable room. Each session lasted about $50 \mathrm{~min}$ and was led by a physiotherapist experienced in the technique. All the recommendations of the Traditional Pilates method were followed in relation to its six principles to carry out the exercise program, namely: centralization, concentration, control, precision, breathing and flow.

Nine exercises (Fig. 2a.1 to a.9) were performed for the main muscle groups with progressions each month. The exercises were initially performed in 1 series of 8 repetitions in the first month. Then they were performed in 2 sets of 10 repetitions in the second month. Finally, they were performed in 3 sets of 8 repetitions in the last month.

Three Swiss ball relaxation exercises were performed in 1 set of $30 \mathrm{~s}$ each (Fig. 2a.10 to a.12) at the end of each session. The exercises were demonstrated and taught to women, but without an adaptation period.

\section{Aquatic aerobic exercise group (AAEG)}

The AAEG patients performed aquatic aerobic exercises at a swimming pool with a temperature of 31 degrees celsius. Each session lasted about $40 \mathrm{~min}$ and was directed by a physiotherapist experienced in water exercises.

The program consisted of six main exercises (Fig. 2b.1 to b.6) lasting $30 \mathrm{~min}$ with different intensity exercises moderated by the Borg scale [33]. Two warm-up exercises and two cool-down exercises were performed before and after the program. Participants were instructed to report subjective perception of respiratory effort during the sessions. The patients reported when it was possible to increase the speed of the exercises in each session.

The exercise programs can be viewed in Fig. 2.

\section{Evaluations}

Both groups were evaluated by blind evaluators who were previously trained for applying the instruments. The evaluations were performed at baseline and 12 weeks after randomization.

\section{Primary outcome}

Pain - visual analogue scale (VAS) Pain intensity was assessed, where 0 is "no pain" and 10 is "the worst pain possible" [31]. A 2-point decrease on a 0-10 pain intensity scale can be considered a clinically important improvement for individual patients [34].

\section{Secondary outcomes}

Disease-related quality of life Disease-related quality of life was assessed by a validated Fibromyalgia Impact 


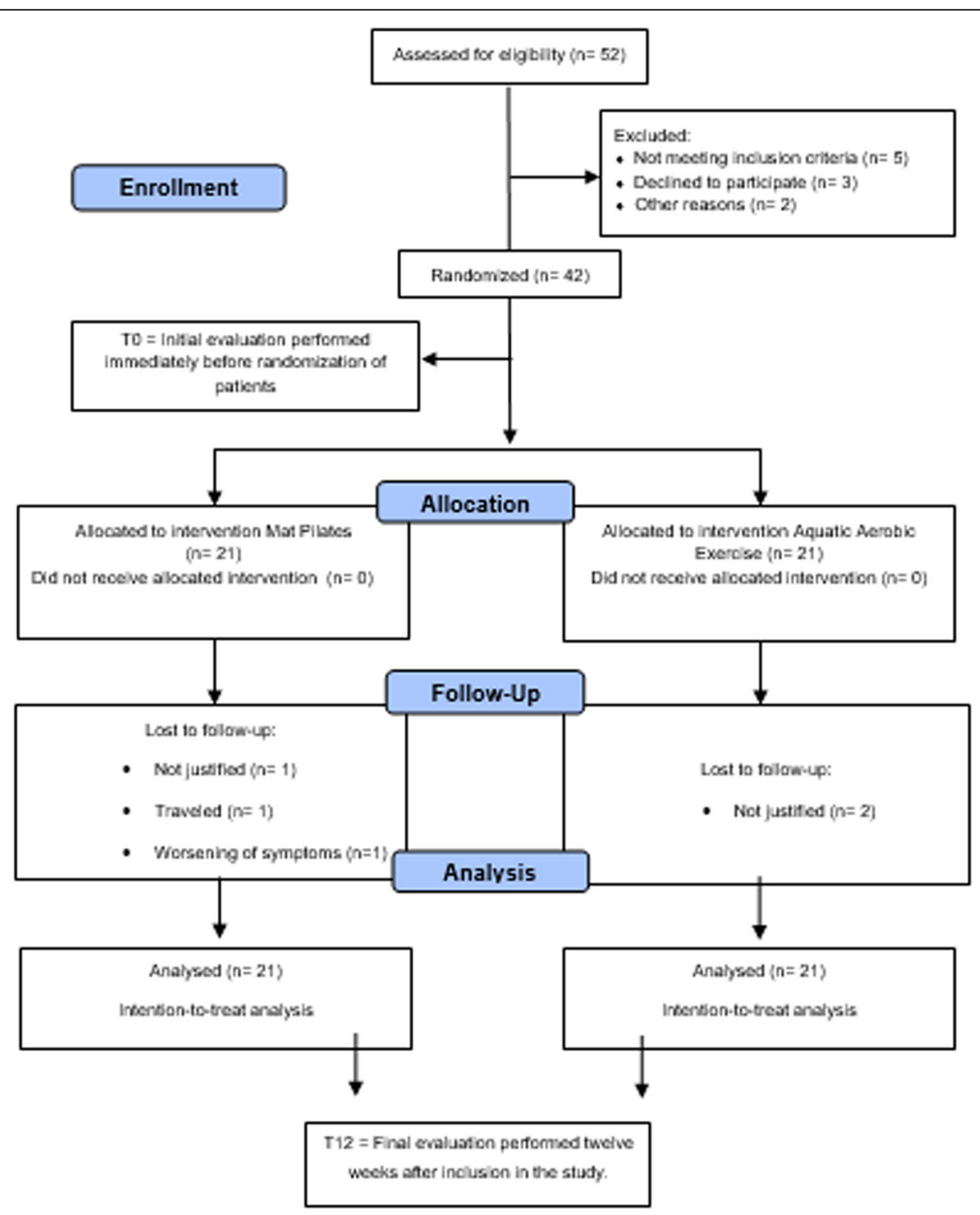

Fig. 1 Flowchart of study

Questionnaire (FIQ) for the Brazilian population. The ten items of the FIQ were normalized to vary from zero to 100. Each item has different scores (the functional capacity item was added and divided by 30 , the two items from zero to 7 divided by seven, and items from zero to 10 divided by 10) [35]. Changes of $14 \%$ change in the total score of the FIQ are clinically relevant [36].

Overall quality of life Quality of life was assessed using the Brazilian version of the Short Form-36 Health Survey (SF-36). This questionnaire consists of eight areas of quality of life: functional capacity, role physical, pain, general health status, vitality, role social, emotional aspects and mental health. Scores range from zero to 100, with a higher score representing better quality of life [37, 38].
Sleep quality Sleep quality was measured by the Brazilian version of the Pittsburgh Sleep Quality Index (PSQI). This instrument consists of 19 questions regarding quality and sleep disorders in the last month. The scores can vary from zero to 3 for each component with the maximum score being 21 points, and scores above 5 indicate poor quality of the individual's sleep [39].

Catastrophic thoughts on pain Catastrophic thoughts on pain were evaluated from the Brazilian version of the Catastrophic Thoughts on Pain Scale (PRCTS), which is composed of 9 items staggered on a Likert scale ranging from zero to 5 points associated with the words "almost never" and "almost always" at the ends. The total score 


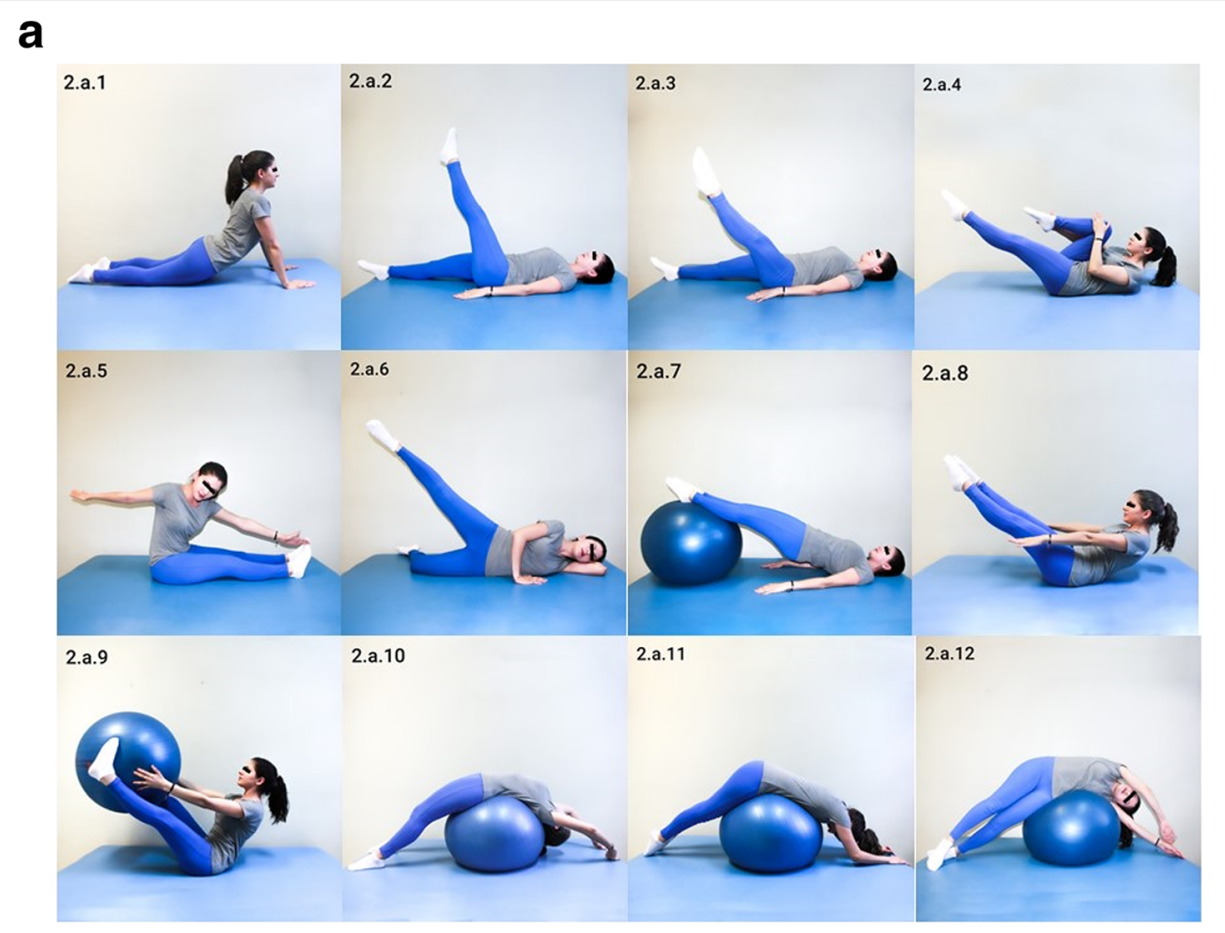

b

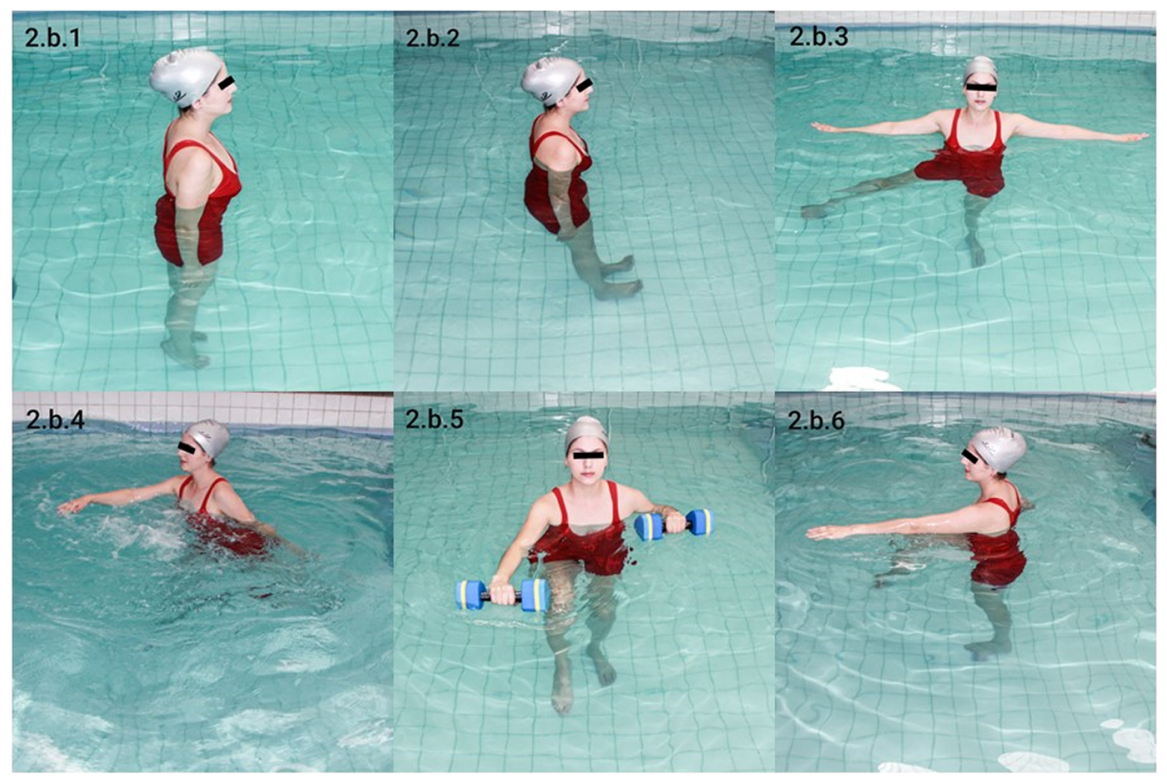

Fig. 2 Exercises program: a.1 to a.12 for Mat Pilates Group and b.1 to b.6 for Aquatic Aerobic Exercise Group

is the sum of the items divided by the number of items answered, with the minimum score being zero and maximum 5. There are no cut-off points, and higher scores indicate greater presence of catastrophic thoughts [40].

Fears and beliefs Fears and beliefs related to physical and occupational activities were evaluated by the Brazilian version of the Fear Avoidance Beliefs Questionnaire (FABQ-
BR). The scale is composed of 16 items, wherein items 1,8 , 13, 14 and 16 are excluded in the sum of the final score. The score must be obtained in isolation in each of the subscales, with the distribution of the points of the subscale related to the work (FABQ Work) done by adding items 6,7 , $9,10,11,12$ and 15 (total ranging from zero to 42 ) and the subscale related to physical activities (FABQ Phys), adding items 2, 3, 4 and 5 (total ranging from zero to 24) [41]. 


\section{Statistical analysis}

Data were analyzed by SPSS software 20.0. The normal distribution and homogeneity of the variances of the variables were verified using the Kolmogorov-Smirnov test and the Levene test, respectively. Estimates of average effect (differences between groups) for all variables were calculated using the ANOVA mixed model. This analysis model incorporated the intervention groups (MPG $\mathrm{x}$ AAEG), time (baseline and 12 weeks) and the group $\times$ time interaction. When a significant $F$ value was found, the Bonferroni post-hoc test was applied in order to identify the differences. The intention-to-treat analysis was used to assess the response to intervention, with the last evaluation repeated as needed. A 95\% confidence interval and a statistical significance level of $5 \%$ were used.

\section{Results}

Some patients dropped-out of the intervention. The MPG presented 3 follow-up losses during the study, while the AAEG presented 2 losses in T12. It is important to emphasize that the performed analysis was of the total sample with an intention-to-treat analysis. Treatment adherence percentage was $85.7 \%$ for the MPG and 90.5\% for the AAEG groups.

Table 1 shows the homogeneity between the groups in the baseline for all analyzed variables.

Table 2 shows the mean and standard deviation values of the analyzed variables in the two evaluated moments (T0 and T12) in the two groups evaluated.

The results for FIQ showed improvement in both groups, with a mean difference $(\mathrm{MD})=0.91,95 \%$ confidence interval $(\mathrm{CI})=0.36 / 1.4,(p=0.002)$ for the AAEG and $\mathrm{MD}=1.695 \% \mathrm{CI}=1.1 / 2.2,(p=0.001)$ for the MPG. In addition, there was also improvement in pain assessed by the VAS, with $\mathrm{MD}=1.8,95 \% \mathrm{CI}=0.74 / 2.8,(p=0.001)$ for the AAEG and MD $=1.3,95 \% \mathrm{CI}=0.22 / 2.3,(p=0.01)$ for the MPG (Table 2).

The MPG showed improvement in the following SF-36 domains: vitality $(\mathrm{MD}=-9.1495 \% \mathrm{CI}=0.06 / 18.2, p=$ $0.04)$, functional capacity $(\mathrm{MD}=-9.5,95 \% \mathrm{CI}=-18.2 /-$ $0.06, p=0.04)$ and pain $(\mathrm{MD}=-11.5,95 \% \mathrm{CI}=-21.0 /-2.0$, $p=0.02)$. In the FABQ-Phys, there was improvement in the activities domain $(\mathrm{MD}=4.9,95 \% \mathrm{CI}=1.6 / 8.2, p=$ $0.005)$. For the AAEG, there were improvements in PSQItotal $(\mathrm{MD}=2.7,95 \% \mathrm{CI}=1.0 / 4.3, p=0.02)$ and in PRCTS $(\mathrm{MD}=0.74,95 \% \mathrm{CI}=0.3 / 1.1, p=0.01)$ (Table 2).

Table 3 presents the analysis between groups at 12 weeks after randomization. No difference was detected between the groups in the performed evaluations for all variables.

\section{Discussion}

The mat Pilates method and the aquatic aerobic exercise in the present study were effective as a form of
Table 1 Mean, standard deviation (SD) values of all analyzed variables of the two groups of patients with fibromyalgia at baseline

\begin{tabular}{llll}
\hline Variable mean (SD) & $\begin{array}{l}\text { AAEG } \\
(\boldsymbol{n}=21) \\
\text { Mean } \pm \text { SD }\end{array}$ & $\begin{array}{l}\text { MPG } \\
(\boldsymbol{n}=21)\end{array}$ & $P$ \\
\hline Age (years) & $50.7(9.7)$ & $45.5(10.6)$ & 0.10 \\
BMI (kg) & $30.4(5.2)$ & $27.8(4.7)$ & 0.10 \\
VAS & $7.5(1.8)$ & $7.5(1.6)$ & 0.86 \\
FIQ & $67(16)$ & $68(14)$ & 0.88 \\
SF36 & & & \\
Role social & $49.5(24.5)$ & $54.2(21.3)$ & 0.51 \\
General health status & $29.7(22.6)$ & $38.2(19.2)$ & 0.20 \\
Vitality & $36.2(18.9)$ & $34.6(17.5)$ & 0.78 \\
Functional capacity & $28.5(16.6)$ & $34.0(17.1)$ & 0.30 \\
Role physical & $17.8(30.7)$ & $23.7(28.8)$ & 0.52 \\
Emotional aspects & $22.2(33.9)$ & $44.4(46.3)$ & 0.08 \\
Pain & $29.4(18.0)$ & $33.3(17.2)$ & 0.47 \\
Mental health & $47.1(22.7)$ & $57.5(21.9)$ & 0.14 \\
PSQI TOTAL & $12.3(4.1)$ & $10.3(3.8)$ & 0.11 \\
PRCTS & $3.04(1.2)$ & $2,64(1.2)$ & 0.29 \\
FABQ_Work & $27.5(12.6)$ & $25.0(10.8)$ & 0.49 \\
FABQ_Phys & $12.6(7.0)$ & $13.0(7.1)$ & 0.83 \\
\hline AAEG Aqutic & & & \\
& & &
\end{tabular}

AAEG Aquatic Aerobic Axercise Group, MPG Mat Pilates Group, BMI Body mass index, VAS Visual analogic pain scale, FIQ Fibromyalgia impact questionnaire, SF36 Short Form-36 Health Survey, PSQI TOTAL Pittsburgh Sleep Quality Index, PRCTS Scale of catastrophic thoughts on pain, FABQ_Work Fear Avoidance Beliefs Questionnaire_Related to work, FABQ_Phys Fear Avoidance Beliefs Questionnaire related to their physical activities

treatment for 12 weeks for women with fibromyalgia, promoting improvement in pain and quality of life. These results were intragroup, without significant differences between the groups. Thus, the strength of this study lies in showing the benefits of two different exercise modalities for women with fibromyalgia. Additionally, fibromyalgia patients may have the option of choosing mat Pilates or aquatic aerobic exercise to improve their symptoms. The mat Pilates presented intragroup results in improving the vitality, functional capacity and pain domains of SF-36, favoring improvement in patients' quality of life, as well as in the field of FABQ-BR activities, which leads to reducing fears and beliefs related to physical activities.

The aquatic aerobic exercise provided positive intragroup results in the improvement of PSQI-total referring to quality of life and sleep quality, and a reduction of catastrophic thoughts towards the disease as evaluated by the PRCTS. Also, there were significant intragroup results in both groups for quality of life in relation to the disease evaluated by the FIQ and in the pain evaluated by the VAS. Furthermore, the groups showed homogeneity for all analyzed variables. 
Table 2 Mean values and standard deviation (SD) of the analyzed variables in the two evaluation moments (T0 and T12) in the two evaluated groups

\begin{tabular}{|c|c|c|c|c|c|c|}
\hline \multirow[t]{3}{*}{ Variables } & \multicolumn{3}{|l|}{$\begin{array}{l}\text { AAEG } \\
(\boldsymbol{n}=21)\end{array}$} & \multicolumn{3}{|l|}{$\begin{array}{l}\text { MPG } \\
(\boldsymbol{n}=21)\end{array}$} \\
\hline & \multicolumn{3}{|l|}{$\begin{array}{l}\text { Mean } \pm \text { SD } \\
(C l \text { 95\%) }\end{array}$} & \multicolumn{3}{|l|}{$\begin{array}{l}\text { Mean } \pm \text { SD } \\
(C l \text { 95\%) }\end{array}$} \\
\hline & T0 & $\mathrm{T} 12$ & $p$ & T0 & $\mathrm{T} 12$ & $p$ \\
\hline VAS & $7.5(1.8)$ & $5.6(2.4)$ & $0.001^{*}$ & $7.5(1.6)$ & $6.2(1.4)$ & $0.01 *$ \\
\hline FIQ & $67(16)$ & $58(16)$ & $0.002^{*}$ & $68(14)$ & $51(17)$ & $0.001 *$ \\
\hline \multicolumn{7}{|l|}{ SF36 } \\
\hline Role social & $49.5(24.5)$ & $53.6(32.3)$ & 0.15 & $54.2(21.3)$ & $64.2(22.1)$ & 0.11 \\
\hline General health status & $29.7(22.6)$ & $37.0(22.3)$ & 0.21 & $38.2(19.2)$ & $39.0(23.6)$ & 0.30 \\
\hline Vitality & $36.2(18.9)$ & $42.6(17.6)$ & 0.16 & $34.6(17.5)$ & $43.8(19.5)$ & $0.04^{*}$ \\
\hline Functional capacity & 28.5 (16.6) & $33.9(18.0)$ & 0.25 & $34.0(17.1)$ & $43.5(22.0)$ & $0.04^{*}$ \\
\hline Role physical & $17.8(30.7)$ & $21.9(32.4)$ & 0.15 & $23.7(28.8)$ & $36.2(38.6)$ & 0.17 \\
\hline Emotional aspects & $22.2(33.9)$ & $34.6(41.2)$ & 0.45 & $44.4(46.3)$ & $43.6(43.6)$ & 0.50 \\
\hline Pain & $29.4(18.0)$ & $37.9(20.3)$ & 0.08 & $33.3(17.2)$ & $44.9(18.4)$ & $0.02^{*}$ \\
\hline Mental health & $47.1(22.7)$ & $55.0(19.3)$ & 0.11 & $57.5(21.9)$ & $65.9(27.8)$ & 0.09 \\
\hline PSQI TOTAL & $12.3(4.1)$ & $9.5(3.7)$ & $0.002^{*}$ & $10.3(3.8)$ & $9.9(3.7)$ & 0.64 \\
\hline PRCTS & $3,04(1.2)$ & $2.3(1.5)$ & $0.001^{*}$ & 2,64 (1.2) & $2.5(1.4)$ & 0.60 \\
\hline FABQ_Work & 27.5 (12.6) & $30.3(11.2)$ & 0.33 & $25.0(10.8)$ & $25.8(14.0)$ & 0.40 \\
\hline FABQ_Phys & $12.6(7.0)$ & $10.9(8.4)$ & 0.32 & $13.0(7.2)$ & $8.1(6.9)$ & $0.005^{*}$ \\
\hline
\end{tabular}

AAEG Aquatic aerobic Exercise Group, MTG Mat Pilates Group, VAS Visual analogue pain scale, FIQ Fibromyalgia impact questionnaire, SF36 Short Form-36 Health Survey, PSQI TOTAL Pittsburgh Sleep Quality Index, PRCTS Scale of catastrophic thoughts on pain, FABQ_Work Fear Avoidance Beliefs Questionnaire_Related to work, FABQ_Phys Fear Avoidance Beliefs Questionnaire related to their physical activities

*Significant difference $(p<0.05)$

Therefore, it is known that the recommendations for practicing physical exercise in treating FM are evident in the literature. The EULAR review (2017) provides a strong recommendation for exercise use, especially because of its effect on pain, physical function and well-being, availability, relatively low cost, and lack of safety concerns. However, it was not possible to distinguish between the aerobic or strength benefits [42].

There is no superior exercise intervention according to Macfarlane et al. [42]. There is also no evidence to suggest superiority of one over another ${ }^{42}$. This corroborates the results presented in our study, in which the MPG did not obtain statistically significant different results when compared to the AAEG, which proves that one modality does not overlap with the other.

According to a study that applied treatment in which the patients were instructed to contract their local musculature ("central stability") before starting any specific exercise on the ground and in water for 6 months presented benefits for improving pain, showing similarity with the findings of our study in the AAEG [3].

The Pilates method is a recommended form of exercise for healthy individuals and for those involved in rehabilitation [43]. In view of this, we believe that the mechanisms which provide these results are those specified in the review by Lima et al. (2017), who propose that there is a balance between inhibition and excitation in the central nervous system, which determines whether exercise promotes analgesia or promotes pain ${ }^{44}$. Several factors, such as physical conditioning level, physical activity levels and state of the injury or pain condition influence this balance [44].

In the study by Altan et al. (2009), the Pilates method as a form of treatment for the intervention group three times a week for 12 weeks achieved significant improvements in VAS values $(p<0.000)$, while the performance frequency of twice a week in the present study was sufficient to obtain significant intragroup results in the VAS values $(p=0.01)[2]$.

The method was also studied in other chronic pain conditions, in which there is evidence that Pilates was more effective than minimal intervention to improve pain, disability, function and overall recovery impression in the short term, and there was no conclusive evidence which showed that it is superior to other forms of exercise [45]. Also, the systematic review by Miyamoto (2013) presents evidence that the exercises based on the Pilates method are more effective than no treatment or minimal intervention in the treatment of chronic nonspecific low back pain [46]. 
Table 3 Differences among T12 assessment groups for all analyzed variables

\begin{tabular}{|c|c|c|}
\hline \multirow[t]{3}{*}{ Variables } & \multicolumn{2}{|c|}{$\begin{array}{l}\text { Differences between groups } \\
\text { Adjusted with mean difference (Cl 95\%) }\end{array}$} \\
\hline & \multicolumn{2}{|l|}{$\begin{array}{l}\mathrm{T} 12 \\
(\mathrm{Cl} 95 \%) . \mathrm{p}\end{array}$} \\
\hline & AAEG VS MPG & $p$ \\
\hline$\overline{\text { VAS }}$ & $-0.61(-1.8$ to 0.6$)$ & 0.61 \\
\hline FIQ & $0.67(-0.37$ to 1.72$)$ & 0.20 \\
\hline \multicolumn{3}{|l|}{ SF36 } \\
\hline Social role & $-10.5(-27.8$ to 6.7$)$ & 0.22 \\
\hline General health status & $-2.0(-16.3$ to 12.2$)$ & 0.77 \\
\hline Vitality & $-1.2(-12.7$ to 10.4$)$ & 0.83 \\
\hline Functional capacity & $-9.6(-22.2$ to 2.9$)$ & 0.13 \\
\hline Physical role & $-14.3(-36.5$ to 7.9$)$ & 0.20 \\
\hline Emotional aspects & $-9.0(-35.5$ to 17.5$)$ & 0.49 \\
\hline Pain & $-7.0(-19.0$ to 5.0$)$ & 0.25 \\
\hline Mental health & $-10.8(-25.7$ to 4.1$)$ & 0.15 \\
\hline PSQI TOTAL & $-0.33(-2.7$ to 2.0$)$ & 0.77 \\
\hline PRCTS & $-0.22(-1.1$ to 0.69$)$ & 0.63 \\
\hline FABQ_Work & $4.4(-3.4$ to 12.3$)$ & 0.26 \\
\hline FABQ_phys & $2.8(-2.0$ to 7.6$)$ & 0.24 \\
\hline
\end{tabular}

AAEG Aquatic aerobic exercise group, MPG Mat Pilates group, VAS Visual analogue pain scale, FIQ Fibromyalgia impact questionnaire, SF36 Short Form36 Health Survey, PSQI TOTAL Pittsburgh Sleep Quality Index, PRCTS Scale of catastrophic thoughts on pain, FABQ_Work Fear Avoidance Beliefs Questionnaire_Related to work, FABQ_Phys Fear Avoidance Beliefs Questionnaire related to their physical activities

For the outcomes found in applying aquatic aerobic exercise, we consider that the mechanisms provided by water immersion are directly related to the present results. These include reduced impact on joints, increased blood flow to muscles and the spread of metabolic waste from muscle to blood, as well as a reduction in the time needed to transport oxygen, nutrients and hormones to the fatigued body [47].

According to Becker (2009), numerous studies of fibromyalgia patients have shown reductions in pain, the impact of fibromyalgia, mood disorders, and improvement in sleep patterns, as well as aquatic groups typically showed faster, more and longer improvements after the study when compared to ground-based exercise programs [48].

In a study conducted by Evcik (2008), the use of the 60-min aquatic therapy performed three times a week for 5 weeks versus the home exercise program obtained significant intragroup results at the 12th week for aquatic therapy, with FIQ having a $p$-value $=$ 0.002 and VAS $p=0.000$. Our study obtained similar results for the AAEG, with values of $p=0.002$ for FIQ and $p=0.001$ for VAS. In addition, Evcik et al. also did not present statistically significant differences between the two groups for FIQ scores, only finding results for VAS $(P<0.001)$ [49]. A Cochrane review (2014) which evaluated sleep through PSQI found moderate evidence of sleep improvement in the group that performed aquatic aerobic exercise [18]. However, better results were obtained in the AAEG in the present study. This is due to the therapeutic properties of heated water, which cause the least possible damage during exercise, providing less impact on the joints, facilitating relaxation, improving muscle tone and microcirculation $[16,20]$.

Pain can directly impact the quality of life of this population. In relation to this outcome, similar findings were found in some studies which reported positive results $[2,16,50]$.

In our study we found significant results in the "activities" domain (FABQ-BR instrument) for the MPG for which it was only an intragroup difference, however in our opinion these results are derived from the principles of centralization, concentration, control, precision, flow and respiration present in the mat Pilates modality.

It is worth remembering that the results of the present study are limited to the studied population and cannot be extrapolated to other populations. Moreover, the study presents some important limitations regarding the small size of the sample and the short execution period, so perhaps more promising results could be observed with the one segment greater than 12 weeks. Also, an important limitation was the comparison of different modalities, loads and intensities imposed on the different systems affected by fibromyalgia. Still, no objective assessment of physical fitness was used in the aquatic aerobic exercise group. The mat Pilates program was performed with great repetition variability between months, and so other studies should focus on comparing different progression protocols. We found some problems in relation to the protocol of this study that was previously published, however we could not reach the sample and the proposed evaluation times as found in the protocol publication. However, even though the obtained results and the effectiveness of the two therapies were positive, it is necessary to conduct new studies with a larger sample number and longer execution time.

\section{Conclusion}

The mat Pilates method and aquatic aerobic exercise were effective after 12 weeks of treatment for patients with FM in improving pain. The MPG did not obtain statistically significant different results when compared to the AAEG, which proves that one modality does not overlap with the other. 


\section{Acknowledgements}

We thank the PROPESQ/UFRN for Internal Financing (numbers 01/2017 - Call for Research Grants of UFRN for the granted scholarships) and we thank all the women with fibromyalgia who participated in this study.

\section{Authors' contributions}

M.C.S., planned the study and drafted the manuscript; S.A.M., R.M.N., and J.B.S.M., collected the data; H.J.A.S., participated in its design and coordination; C.A.A.L., performed the statistical analysis. All authors read and approved the final manuscript

\section{Funding}

"This study was partly financed by the Coordenação de Aperfeiçoamento de Pessoal de Nível Superior - Brasil (CAPES) - Master's degree scholarship, Finance Code 001."

\section{Availability of data and materials}

We declare that the data collected during this research is found in databases of Excel and SPSS software. They are currently in the possession of responsible researchers who undertake to make them available to third parties, if requested. To do so, we kindly ask you to send the request email to: marcelocardoso@facisa.ufrn.br.

\section{Ethics approval and consent to participate}

This study was approved by the Ethics Committee of Federal University of Rio Grande do Norte, Faculty of Health Sciences of Trairi - (FACISA/ UFRN). The ethical principles agreed in the Declaration of Helsinki will be respected for all study procedures. Respect for individuals will be insured and their autonomy will be maintained. Participants will be informed of the study objectives, its risks and benefits. Participants will be free to abandon the study at any time without the obligation of giving any explanation. Participants must sign the informed consent before the study begins.

\section{Consent for publication}

Not applicable.

\section{Competing interests}

The authors declare that they have no competing interests.

\section{Author details \\ ${ }^{1}$ Federal University of Rio Grande do Norte, Faculty of Health Sciences of Trairi - (UFRN/FACISA), Santa Cruz, RN, Brazil. ${ }^{2}$ Federal University of Rio Grande do Norte, Faculty of Health Sciences of Trairi - (UFRN/FACISA), Postgraduate Program in Rehabilitation Sciences, Vila Trairi st, S/N, Centro, Santa Cruz, RN 59200-000, Brazil.}

Received: 15 November 2019 Accepted: 25 February 2020

Published online: 06 April 2020

\section{References}

1. Latorre Román P, Aparecida Santos M, Heredia-Jimenez J, et al. Effect of a 24-week physical training programme (in water and on land) on pain, functional capacity, body composition and quality of life in women with fibromyalgia. Clin Exp Rheumatol. 2013;31:72-80.

2. Altan L, Korkmaz N, Bingol Ü, et al. Effect of Pilates training on people with fibromyalgia syndrome: a pilot study. Arch Phys Med Rehabil. 2009;90:1983-8.

3. Rivas Neira S, Pasqual Marques A, Pegito Pérez I, et al. Effectiveness of aquatic therapy vs land-based therapy for balance and pain in women with fibromyalgia: a study protocol for a randomised controlled trial. BMC Musculoskelet Disord. 2017;18:22.

4. Wolfe F, Smythe HA, Yunus MB, et al. The American College of Rheumatology 1990 criteria for the classification of fibromyalgia. Report of the multicenter criteria committee. Arthritis Rheum. 1990;33:160-72.

5. Wolfe F, Clauw DJ, Fitzcharles MA, et al. The American College of Rheumatology preliminary diagnostic criteria for fibromyalgia and measurement of symptom severity. Arthritis Care Res. 2010;62:600-10.

6. Wolfe F, Clauw DJ, Fitzcharles MA, et al. 2016 Revisions to the 2010/2011 fibromyalgia diagnostic criteria. Semin Arthritis Rheum. 2016;46:319-29.
7. Marques AP, do Espírito Santo AD, Berssaneti AA, et al. Prevalence of fibromyalgia: literature review update. Rev Bras Reumatol (English Ed). 2017; 57:356-63.

8. Häuser W, Fitzcharles MA. Facts and myths pertaining to fibromyalgia. Dialogues Clin Neurosci. 2018;20(1):53-62.

9. Hadlandsmyth K, Dailey DL, Rakel BA, et al. Somatic symptom presentations in women with fibromyalgia are differentially associated with elevated depression and anxiety. J Health Psychol. 2017;1359105317736577. https:// doi.org/10.1177/1359105317736577.

10. Roger-Silva D, Natour J, Moreira E, et al. A resistance exercise program improves functional capacity of patients with psoriatic arthritis: a randomized controlled trial. Clin Rheumatol. 2018;37:389-95.

11. Valim V, Natour J, Xiao Y, et al. Effects of physical exercise on serum levels of serotonin and its metabolite in fibromyalgia: a randomized pilot study. Rev Bras Reumatol (English Ed). 2014;53:538-41.

12. Assunção Júnior JC, de Almeida Silva HJ, da Silva JFC, et al. Zumba dancing can improve the pain and functional capacity in women with fibromyalgia. J Bodyw Mov Ther. 2018;22:455-9.

13. Silva HJA, Assunção Júnior JC, de Oliveira FS, et al. Sophrology versus resistance training for treatment of women with fibromyalgia: $A$ randomized controlled trial. J Bodyw Mov Ther. 2019;23(2):382-89. https://doi.org/10.1016/j.jbmt.2018.02.005.

14. Busch AJ, Barber KAR, Overend TJ, et al. Exercise for treating fibromyalgia syndrome. Cochrane Database Syst Rev. 2007:CD003786.

15. Carville SF, Arendt-Nielsen S, Bliddal H, et al. EULAR evidence-based recommendations for the management of fibromyalgia syndrome. Ann Rheum Dis. 2008;67:536-41.

16. Fernandes G, Jennings F, Nery Cabral MV, et al. Swimming improves pain and functional capacity of patients with fibromyalgia: a randomized controlled trial. Arch Phys Med Rehabil. 2016:97:1269-75.

17. López-Rodríguez MDM, Castro-Sánchez AM, Fernández-Martínez M, et al. Comparación entre biodanza en medio acuático y stretching en la mejora de la calidad de vida y dolor en los pacientes con fibromialgia. Aten Primaria. 2012:44:641-50.

18. Bidonde J, Busch AJ, Webber SC, et al. Aquatic exercise training for fibromyalgia. Cochrane Database Syst Rev. 2014:CD011336.

19. Penelope L. Updating the principles of the Pilates method-part 2. J Bodyw Mov Ther. 2002;6:94-101.

20. Natour J, Cazotti LDA, Ribeiro LH, et al. Pilates improves pain, function and quality of life in patients with chronic low back pain: a randomized controlled trial. Clin Rehabil. 2015;29:59-68.

21. Cruz-Díaz D, Romeu M, Velasco-González C, et al. The effectiveness of 12 weeks of Pilates intervention on disability, pain and kinesiophobia in patients with chronic low back pain: a randomized controlled trial. Clin Rehabil. 2018;32:1249-57.

22. Posadzki P, Lizis P, Hagner-Derengowska M. Pilates for low back pain: a systematic review. Complement Ther Clin Pract. 2011;17:85-9.

23. de Araujo Cazotti L, Jones A, Roger-Silva D, et al. Effectiveness of the Pilates method in the treatment of chronic mechanical neck pain: a randomized controlled trial. Arch Phys Med Rehabil. 2018;99:1740-6.

24. Ekici G, Unal E, Akbayrak T, et al. Effects of active/passive interventions on pain, anxiety, and quality of life in women with fibromyalgia: randomized controlled pilot trial. Women Health. 2017:57:88-107.

25. Wang C, Schmid CH, Fielding RA, et al. Effect of tai chi versus aerobic exercise for fibromyalgia: comparative effectiveness randomized controlled trial. BMJ. 2018;360:k851.

26. Assis MR, Silva LE, Barros Alves AM, et al. A randomized controlled trial of deep water running: clinical effectiveness of aquatic exercise to treat fibromyalgia. Arthritis Care Res. 2006;55:57-65.

27. Begg C, Cho M, Eastwood S, et al. Improving the quality of reporting of randomized controlled trials: the CONSORT statement. J Am Med Assoc. 1996:276:637-9.

28. Yamato T, Maher C, Saragiotto B, et al. The TIDieR checklist will benefit the physiotherapy profession. Physiother Theory Pract. 2017;33:267-8.

29. Silva HJA, Lins CAA, Nobre TTX, de Sousa VPS, Caldas RTJ, de Souza MC. Mat Pilates and aquatic aerobic exercises for women with fibromyalgia: a protocol for a randomised controlled blind study. BMJ Open. 2019;9(2): e022306. https://doi.org/10.1136/bmjopen-2018-022306.

30. Bennett RM, Friend R, Marcus D, et al. Criteria for the diagnosis of fibromyalgia: validation of the modified 2010 preliminary American college 
of rheumatology criteria and the development of alternative criteria. Arthritis Care Res. 2014;66:1364-73.

31. Farrar JT, Young JP, LaMoreaux L, et al. Clinical importance of changes in chronic pain intensity measured on an 11-point numerical pain rating scale. Pain. 2001;94:149-58.

32. Gustafsson M, Gaston-Johansson F. Pain intensity and health locus of control: a comparison of patients with fibromyalgia syndrome and rheumatoid arthritis. Patient Educ Couns. 1996;29:179-88.

33. Shariat A, Cleland JA, Danaee $M$, et al. Borg CR-10 scale as a new approach to monitoring office exercise training. Work. 2018;60:549-54.

34. Dworkin RH, Turk DC, McDermott MP, et al. Interpreting the clinical importance of group differences in chronic pain clinical trials: IMMPACT recommendations. Pain. 2009;146:238-44.

35. Marques AP, Santos AMB, Assumpção A. Validação da Versão Brasileira do Fibromyalgia Impact Questionnaire (FIQ) Validation of the Brazilian version of the Fibromyalgia Impact Questionnaire (FIQ)]. Medicina (B Aires). 2006: 24-31.

36. Bennett RM, Bushmakin AG, Cappelleri JC, et al. Minimal clinically important difference in the fibromyalgia impact questionnaire. J Rheumatol. 2009;36: 1304-11.

37. Bottoli I, Beharry K, Modanlou HD, et al. Effect of group B streptococcal meningitis on retinal and choroidal blood flow in newborn pigs. Investig Ophthalmol Vis Sci. 1995;36:1231-9.

38. Guimarães LH d CT, Galdino DCA, Martins FLM, et al. Avaliação da capacidade funcional de idosos em tratamento fisioterapêutico. Revista Neurociências. 2004:130-3.

39. Passos MHP, Silva HA, Pitangui ACR, et al. Reliability and validity of the Brazilian version of the Pittsburgh Sleep Quality Index in adolescents. Pediatr (Versão em Port). 2017;93:200-6.

40. Sardá J, Nicholas MK, Pereira IA, et al. Validação da Escala de Pensamentos Catastróficos sobre Dor. Acta Fisiatria. 2008;15:31-6.

41. Abreu AM, Faria CD, Cardoso SM, et al. Versão brasileira do Fear Avoidance Beliefs Questionnaire. Cad Saúde Pública. 2008;24(3):615-23.

42. Macfarlane GJ, Kronisch C, Dean LE, et al. EULAR revised recommendations for the management of fibromyalgia. Ann Rheum Dis. 2017:76(2):318-28.

43. Patti A, Bianco A, Paoli A, et al. Effects of Pilates exercise programs in people with chronic low back pain: a systematic review. Medicine (United States). 2015;94:22-5.

44. Lima LV, Abner TSS, Sluka KA. Does exercise increase or decrease pain? Central mechanisms underlying these two phenomena. J Physiol. 2017;595: 4141-50.

45. Yamato TP, Maher CG, Saragiotto BT, et al. Pilates for low back pain: complete republication of a Cochrane review. Spine. 2016;41:1013-21.

46. Miyamoto GC, Costa LOP, Cabral CMN. Efficacy of the Pilates method for pain and disability in patients with chronic nonspecific low back pain: a systematic review with meta-analysis. Braz J Phys Ther. 2013;17:517-32.

47. Torres-Ronda L, Schelling I, Del Alcázar X. The properties of water and their applications for training. J Hum Kinet. 2014;44:237-48.

48. Becker BE. Aquatic therapy: scientific foundations and clinical rehabilitation applications. PM R. 2009;1:859-72

49. Evcik D, Yigit I, Pusak H, et al. Effectiveness of aquatic therapy in the treatment of fibromyalgia syndrome: a randomized controlled open study. Rheumatol Int. 2008;28:885-90.

50. Sosa-Reina MD, Nunez-Nagy S, Gallego-Izquierdo T, et al. Effectiveness of therapeutic exercise in fibromyalgia syndrome: a systematic review and meta-analysis of randomized clinical trials. Biomed Res Int. 2017;2017:1-14.

\section{Publisher's Note}

Springer Nature remains neutral with regard to jurisdictional claims in published maps and institutional affiliations.

Ready to submit your research? Choose BMC and benefit from:

- fast, convenient online submission

- thorough peer review by experienced researchers in your field

- rapid publication on acceptance

- support for research data, including large and complex data types

- gold Open Access which fosters wider collaboration and increased citations

- maximum visibility for your research: over $100 \mathrm{M}$ website views per year

At BMC, research is always in progress.

Learn more biomedcentral.com/submissions 